\title{
Didáctica disruptiva STEM
}

Cambiando el paradigma de la docencia tradicional a la docencia coaching
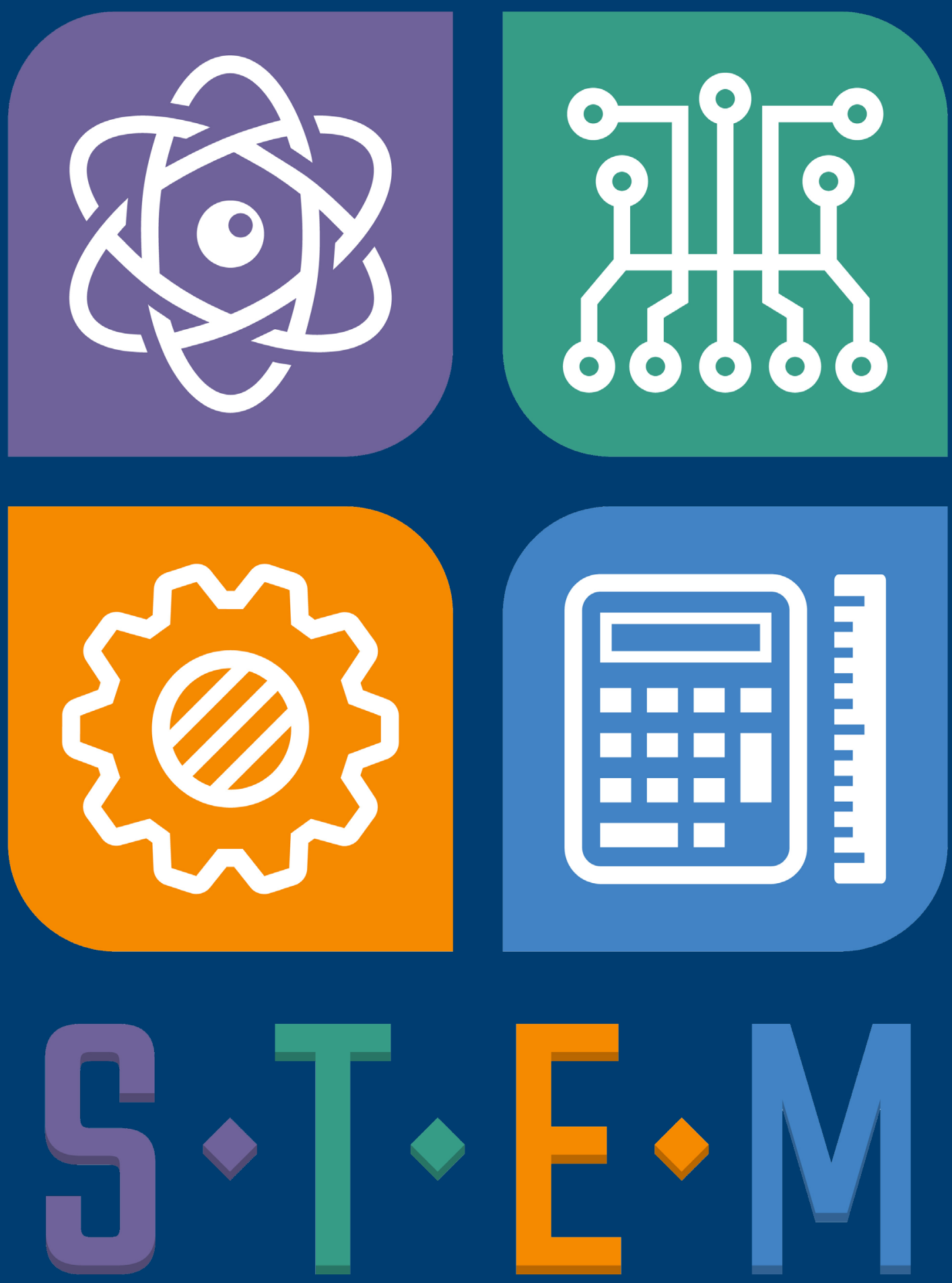


\title{
Didáctica disruptiva STEM. Cambiando el paradigma de la docencia tradicional a la docencia coaching
}

\author{
Melvin E. Chaves Duarte \\ mchaves@ufidelitas.ac.cr, Universidad Fidélitas
}

\begin{abstract}
The authorities of the Fidélitas University of Costa Rica, have established as a strategic project, the search for a contemporary and disruptive curricular model, to respond to the new generations of creative students of the XXI century.
\end{abstract}

This model is adopted from the curricular analysis of the main Universities worldwide, such as Harvard University, the Massachusetts Institute of Technology (MIT), the Federal University of Zurich (ETH) and the National University of Singapore (NUS), universities that are in the first places of the QS ranking of England, for their capacity for innovation to solve the global problems of the next 50 years.

The curricular model adapted by the Fidélitas University is based on disruptive STEM didactics (whose acronym stands for Science, Technology, Engineering \& Mathematics). The National Science Foundation proposed the STEM model in the early 1990s. In this regard, it is said that science (S) and mathematics (M) provide research and measurement, then engineering (E) designs and innovate in the search for the solution of real problems and as a result, the technology (T) is obtained, which refers to the products, services and systems, provided by Engineering in the solution of complex problems. At the same time, it is an integrating curricular approach: it integrates knowledge, to know how to know, to know how to do, to know how to be and to know how to live together, fundamental educational pillars of UNESCO (Brodeur, 2007).

The philosophy of the STEM model, enhances the creativity of students and teachers to solve complex problems, in order to achieve continuous improvement of society, turning needs into creative ideas necessary to engage generations of digital students ( $\mathrm{Y}$ - Millennials) and virtual ( $\mathrm{Z}$ and Alpha).

Keywords: STEM Curriculum, Teacher Coaching, disruptive didactics, exponential education, Millennials generation, Generation Z, Generation Alpha, Desing Thinking.

\section{Resumen}

Las autoridades de la Universidad Fidélitas de Costa Rica, han establecido como un proyecto estratégico, la búsqueda de un modelo curricular contemporáneo y disruptivo, para responder a las nuevas generaciones de estudiantes creativos del Siglo XXI. Este modelo se adopta del análisis curricular de las principales Universidades a nivel mundial, como la Universidad de Harvard, el Instituto Tecnológico de Massachusetts (MIT), la Universidad Federal de Zúrich (ETH) y la Universidad Nacional de Singapur (NUS), universidades que ocupan los primeros lugares del ranking QS de Inglaterra, por su capacidad de innovación para resolver los problemas globales de los próximos 50 años.

El modelo curricular adaptado por la Universidad Fidélitas se fundamenta en la didáctica disruptiva STEM (cuyas siglas significan Science, Technology, Engineering \& Mathematics), esto se traduce como ciencia, tecnología, ingeniería y matemática. La National Science Foundation propuso el modelo STEM, a inicios de la década de los $90 . \mathrm{Al}$ respecto, se dice que la ciencia (S) y la matemática (M) aportan la investigación y la medición, para que la ingeniería (E) diseñe e innove en la búsqueda de la solución de problemas reales y como resultado, se obtiene la tecnología (T), la cual se refiere a los productos, servicios y sistemas, aportados por la Ingeniería en la solución de los problemas complejos. Al mismo tiempo, es un enfoque curricular integrador: integra el saber saber, el saber hacer, el saber ser y el saber convivir, pilares fundamentales educativos de la UNESCO (Brodeur, 2007).

La filosofía del modelo STEM, potencia la creatividad de los estudiantes y los docentes para resolver problemas complejos, con el fin de lograr la mejora continua de la sociedad, convirtiendo las necesidades en ideas creativas necesarias para enganchar a las generaciones de los estudiantes digitales (YMillennials) y virtuales $(Z$ y Alfa $)$.

Palabras clave: Currículo STEM, Coaching Docente, didáctica disruptiva, educación exponencial, generación Millennials, Generación Z, Generación Alfa, Desing Thinking. 


\section{Introducción}

El propósito de este artículo es analizar desde la perspectiva teórica el modelo curricular STEM, para responder a las necesidades didácticas de los docentes y los estudiantes de las carreras de Ingenierías, Ciencias Sociales y Económicas de la Universidad Fidélitas.

La filosofía STEM, se fundamenta en la disrupción, esta teoría hace referencia a una "rotura o interrupción brusca". (Pérez, 2017).

Podemos decir entonces, que, al hablar de didáctica disruptiva STEM, hacemos referencia a una forma de aprender, que rompe con lo establecido, interrumpiendo el tradicional modelo de transmisión de conocimientos. En el marco anterior, la didáctica disruptiva STEM, tiene sus bases epistemológicas en la investigación desarrollada por el Dr. Clayton Christensen de la Universidad de Harvard, sobre disrupción educativa. Esta teoría según el Dr. Christensen garantiza la actividad intelectual y creativa de los estudiantes y los docentes, ya que se focaliza en el proceso formativo (Coaching Docente), donde el profesor como el estudiante, son actores de un todo, que se encuentran en una perpetua sinergia de creatividad e innovación exponencial para la resolución de problemas reales del contexto.

En el Siglo XXI la creatividad para resolver problemas reales complejos se ha convertido en una destreza que todo empleador desea en sus colaboradores para lograr una innovación abierta en la organización. Un profesional que desarrolle esta competencia convertirá las necesidades de la sociedad en ideas creativas (CINDE,2016).

Actualmente en la Universidad Fidélitas convergen la generación de los Millennials de 17-34 años (nacidos entre 1982 y1999), que tiene como decisión de vida el formarse y trabajar por la pasión que les mueve a resolver problemas a través del emprendedurismo, el sentido social, la ética y el respeto al medio ambiente y la nueva generación, llamada los Z y los Alpha de 0 a 20 años (nacidos 2000-2020), conocidos como la población sedentaria virtual, que se caracterizan por el consumo de dispositivos electrónicos, como los videojuegos, las aplicaciones y las plataformas virtuales (Vilanova,2017).

El modelo educativo STEM considera las características de la generación creativa (Millennials, los Z y los Alpha) mediante el diseño de metodologías abiertas que permiten cuantificar las acciones de innovación y cambio, y tomar decisiones inteligentes para mejorar la cognición de estas generaciones de estudiantes, utilizando los métodos de investigación aplicada y el pensamiento estratégico de diseño (Desing Thinking) para resolver los problemas de la sociedad contemporánea (Villardon, 2013).

\section{Educación en la era del conocimiento}

En la era del conocimiento encontramos continuos cambios que se producen a gran velocidad y que afectan de forma significativa a la sociedad, pero, paradójicamente la educación permanece inamovible, en el sistema educativo nada parece haber cambiado. La era del conocimiento ha traído avances con los cuales podemos construir y crear innovaciones. La inteligencia artificial, la robótica, los drones, el reconocimiento facial, apps inteligentes, realidad virtual y aumentada, transportes inteligentes, naves no tripuladas, internet de las cosas.... este es el mundo del Siglo XXI, entonces por qué seguimos con aulas y métodos de enseñanza victorianos del Siglo XVIII?

El modelo STEM se instrumenta en el espacio áulico abierto, a través de una metodología didáctica disruptiva, que transformará la personalidad de los estudiantes, convirtiéndolos en personas críticas y analíticas.

El sistema educativo, ha creado estudiantes que no saben resolver problemas, que han estudiado las matemáticas y las ciencias de memoria para aprobar los exámenes, esto se considera, una amputación de la creatividad y la innovación (Robinson, K. 2016).

Entonces cómo hacer para que un proceso de aprendizaje se vuelva rápido y eficaz, esto se logrará, con el desarrollo de una estructura aprendizaje STEM, donde las habilidades creativas se integren para resolver problemas, para esto los profesores y los estudiantes deben cambiar el "Chip" y salir de zona de confort.

Para la adaptación del modelo STEM en la Universidad Fidélitas, se realizó cinco talleres participativos de didáctica disruptiva, con setenta expertos del Claustro de profesores de Ingeniería, Derecho, Administración, Ingeniería en Sistemas Computacionales, para tener una idea acertada del modelo STEM y su aplicación práctica en las aulas, tanto virtuales como presenciales de la Universidad.

\section{EI desarrollo del modelo STEM en los sistemas universitarios.}

Según estudio de referentes realizados por área curricular de la Universidad Fidélitas, en la Universidad de Harvard, el modelo STEM moviliza e integra los conocimientos, habilidades, actitudes y valores, hacia la consecución de propósitos concretos; son más que el saber, el saber hacer o el saber ser, porque se manifiestan en la acción de manera exponencial, son competencias para la vida que deberán proporcionar oportunidades y experiencias de aprendizaje significativas para todos los estudiantes, de ésta manera se desarrolla el aprendizaje permanente, el manejo de la información, el manejo de situaciones complejas y la convivencia.

Para el Instituto Tecnológico de Massachusetts (MIT) el modelo STEM se relaciona estratégicamente con la investigación para la resolución de problemas reales, a 
través del desarrollo de destrezas de comunicación asertiva, de trabajo en equipo y de emprendimiento.

La Universidad Federal de Zúrich (ETH) promueve el modelo STEM, focalizado en resolver problemas reales, considerando los métodos y técnicas investigativas aplicada para el trabajo.

Finalmente, la Universidad de Singapur desarrolla un enfoque de aprendizaje STEM e investigación aplicada. La calidad educativa, unida a la investigación de alto impacto y al concepto de la universidad como servicio público para la sociedad, preside sus actuaciones. El modelo pedagógico STEM se fundamenta en competencias para la resolución de problemas reales del modelo Triple Hélix (EmpresaGobierno-Sociedad)

El proyecto académico STEM, de la Universidad Fidélitas se fundamenta en la articulación de una comunidad de aprendizaje, cuyo principal objetivo es garantizar que el estudiante desarrolle al máximo su potencial creativo y que, durante su estancia en este centro, transite por una experiencia de formación integral, para resolver problemas reales.

El proceso de Bolonia y el Proyecto Tuning (Innovación educativa y social), ha enfatizado el desarrollo de competencias (Vásquez García, 2011), y ha recomendado a los países miembros, propuestas didácticas transformadoras bajo el enfoque curricular STEM. (Corominas, 2006).

Lo esencial del concepto es que las competencias STEM están vinculadas a la acción, esto es, deben realizarse y actuarse en un contexto real determinado, integrando los diferentes elementos (saberes, destrezas, habilidades y actitudes) y favorecen la solución de problemas profesionales y sociales (Villardon, 2013).

\section{EI modelo curricular STEM de la Universidad Fidélitas}

El modelo curricular STEM de la Universidad Fidélitas fue validado por un equipo interdisciplinario de docentes, que durante varios años han formado profesionales, en las áreas de Ciencias Empresariales, Ingeniería y Ciencias Sociales. El modelo está concebido para formar estudiantes creativos e innovadores, que les permitan desempeñarse de manera eficaz y además, para ser buenos ciudadanos con valores éticos y de responsabilidad ambiental y social, capaces de afrontar las situaciones presentes y futuras, como eje principal de la educación.

En la Figura 1, se muestra gráficamente el modelo STEM de la Universidad, se puede observar que, en el centro del mismo existe la integración de la ciencia, la tecnología, las matemáticas y la ingeniería. El estudiante desarrollará sus conocimientos, habilidades y actitudes para aprender en forma integral y real. El modelo pretende lograr un aprendizaje que sitúe a cada estudiante en las mejores condiciones educativas posibles, para que continúe aprendiendo durante toda su vida. La rapidez del cambio que atraviesa el ámbito social y profesional exige que estén preparados para dirigir y regular su propio aprendizaje, porque será un proceso continuo de prueba y error, con esto el estudiante y el profesor pierden el miedo a la equivocación, considerándola como un aprendizaje de mejora continua.

El círculo uno del modelo, es el punto de partida del aprendizaje STEM, ya que constituye la concepción epistemológica, de que el verdadero aprendizaje es una construcción crítica de cada estudiante que logra modificar su estructura mental, alcanzar un mayor nivel de diversidad, de complejidad y de integración aplicando esquemas de pensamiento complejo y movilización de los conocimientos y de las informaciones.

El círculo dos, es el desarrollo de la persona, al tener las habilidades y los atributos personales y profesionales se evita la fragmentación, esto facilita la integración de contenidos aplicables y la interdisciplinariedad. Se generan aprendizajes situacionales complejos, individuales y en grupo, que favorecen la autonomía de los estudiantes, procura el pensamiento complejo, la innovación y la aplicación de las tecnologías.

En el círculo tres, los estudiantes obtienen las herramientas para convertirse en personas que aprenden a aprender, a ser ciudadanos creativos, innovadores, abiertos y con pensamiento complejo que los prepara para pensar críticamente, anticipar y resolver problemas, priorizar $\mathrm{y}$ negociar, comunicarse asertivamente en forma oral $\mathrm{y}$ escrita, perseverar e integrar soluciones ante las situaciones de la vida.

Finalmente, el círculo 4, enmarca una serie de elementos que integran una actividad observable y sistémica, ya que registra el desempeño de los estudiantes dentro de su entorno y contexto real, con base en la aplicación de un significado o significados de aprendizaje, construidos a través de sus propias experiencias.

En esta dimensión del modelo se fundamentan las acciones reflexivas e integrales que responden a los problemas del contexto, con idoneidad y compromiso ético, integrando el saber ser, el saber hacer y el saber conocer en una perspectiva de mejora continua. Esto incluye la concepción y apropiación de conocimientos, las herramientas de diseño, la implementación y la operación de modelos denominada CDIO, ver Figura 2. 
Figura 1. Modelo curricular STEM Universidad Fidélitas de Costa Rica.

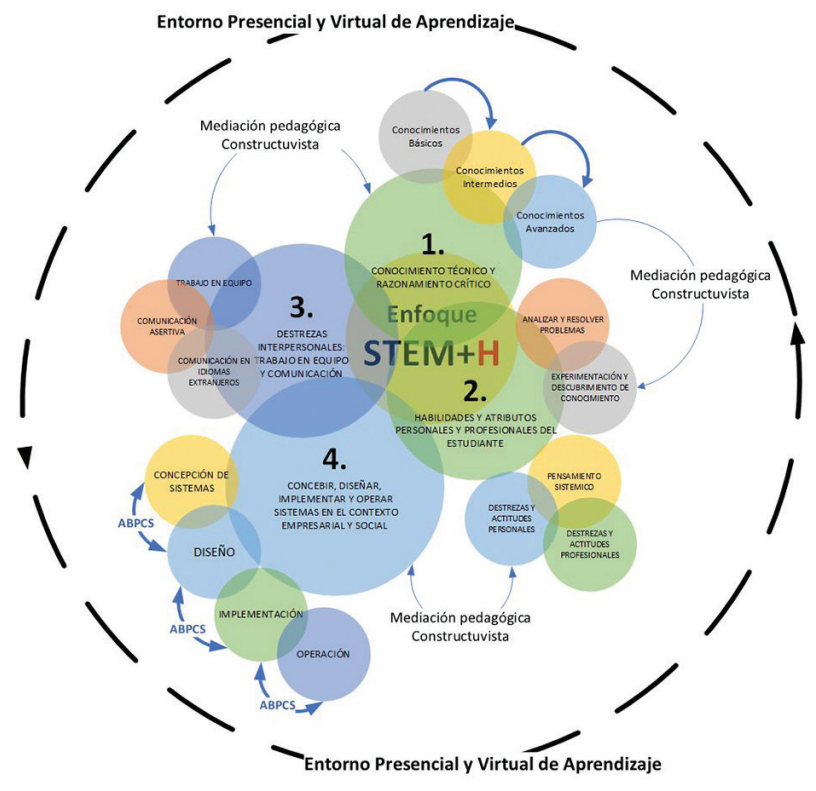

Fuente propia: Desarrollo e innovación Curricular

Figura 2. Etapas para desarrollar proyectos mediante el proceso de CDIO/ABPSC-STEM.

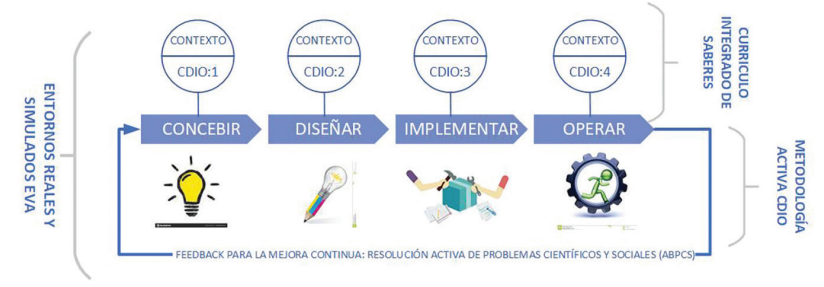

Fuente propia: Desarrollo e innovación Curricular

\section{Resultados}

\section{La fábrica de herramientas didácticas disruptivas: el caso de la Universidad Fidélitas}

Para implementar el modelo STEM la Universidad, ha realizado esfuerzos de pedagogía experimental para el apoyo didáctico de los docentes de las carreras de Ingeniería de Sistemas Computacionales, Ciencias Administrativas, Contaduría Pública, Ingeniería Civil, Derecho; donde se han producido cursos bajo el modelo STEM, con el estándar global setenta por ciento práctico y treinta por ciento teórico (70:30).

Durante los talleres de didáctica disruptiva STEM, se han diseñado espacios de aprendizaje experimentales, considerando las siguientes herramientas didácticas:

a. Aprendizaje basado en proyectos científicos y sociales CDIO/ABPCS-STEM, se define como una metodología activa y colaborativa, que propone proyectos interdisciplinares entre las ciencias sociales y exactas, la tecnología, la ingeniería y las matemáticas, aprovechando los elementos comunes entre ellas, mediante su instrumentalización integral en la resolución de un problema o elaboración de un producto del contexto real o simulado. Ver Figura 1.

Los alumnos en grupo, de forma autónoma y asesorados por el docente, deben encontrar la respuesta a una pregunta o problema, de forma que el conseguir hacerlo correctamente suponga tener que buscar, comprender e integrar los conocimientos básicos, intermedios y avanzados de las asignaturas (Benito y Cruz, 2016).

b. Aprendizaje basado en juegos y gamificación (Second Life). La gamificación es la aplicación de principios y elementos propios del juego en un ambiente de aprendizaje, con el propósito de influir en el comportamiento, incrementar la motivación y favorecer la participación de los estudiantes. La Plataforma utilizada para gamificar fue Second Life (https://secondlife.com/destinations/learning), los profesores simularon espacios de aprendizaje y experimentaron la inmersión en mundo virtual en 3D de más de 15 millones de usuarios.

c. Coaching Docente; Aprendizaje en aula invertida (Flipped learning). El Flipped learning es una estrategia de aprendizaje disruptiva que transfiere el tiempo invertido en dar clases magistrales tradicionales al aprendizaje fuera del aula y optimiza exponencialmente el tiempo de clase, junto con la experiencia del profesor, para desarrollar un coaching docente y potenciar otros procesos de adquisición y práctica de conocimientos dentro del aula presencial o virtual. Cuando los docentes diseñan su clase en un entorno virtual de aprendizaje (EVA), el tiempo de clase se libera para que se pueda facilitar la participación de los estudiantes en el aprendizaje activo y de esta manera resolver problemas complejos (Santiago, R; Bergmann, J. 2018).

d. Estrategias de aprendizaje STEM en Teams for Education (Configuración, hiperconectividad y retroalimentación).

Aulas de Flipped learning, los docentes asesores o coaching y los alumnos participan como miembros de la comunidad aprendizaje. Las aulas Flip de clase permiten crear espacios de aprendizaje colaborativo en tiempo real, registrar comentarios de los alumnos y darles un espacio privado para su desarrollo, según las brechas de aprendizaje diagnosticadas por el docente.

Portafolio didáctico de clase de OneNote es un portafolio de notas digital (PoDi), en el que todos los estudiantes pueden almacenar documentos de texto, 
imágenes, notas escritas a mano, datos adjuntos, vínculos, archivos de voz y vídeo, fórmulas, casos, prácticas, ejercicios, etc.

Cada portafolio de notas está organizado en tres partes:

- Blocs de notas de estudiante: es un espacio privado que comparte el profesor con cada estudiante. Los profesores pueden acceder a todos los blocs de notas de estudiante, pero los estudiantes solo pueden ver el propio.

- Espacio de colaboración: es un espacio didáctico en el que toda la clase puede compartir, organizar y colaborar.

- Biblioteca de contenido: es un espacio de solo lectura en el que los profesores pueden compartir documentos con los estudiantes.

A continuación, se presenta en la Figura 3 un ejemplo de clase invertido, sobre esfuerzos de tensión, donde los estudiantes resolvieron los problemas fuera del aula y evacuaron sus dudas en el espacio de clase sincrónica.

Figura 3. Ejercicio de Simulación en aula invertida sobre esfuerzos de tensión utilizando un PoDi en Teams para Educación.

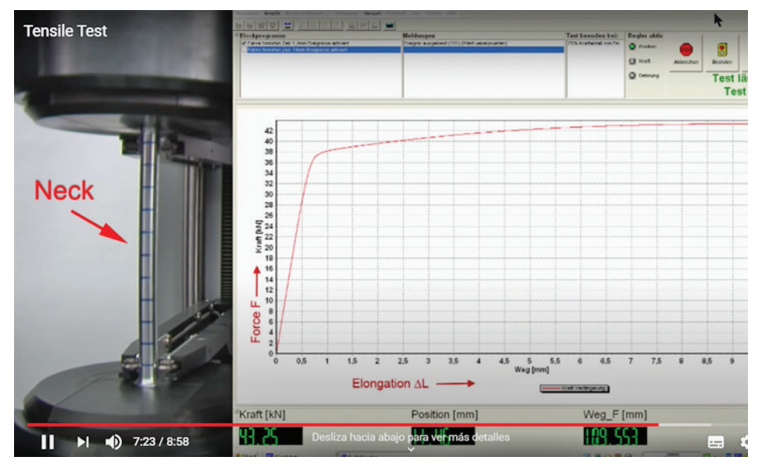

Fuente: Curso de esfuerzo de tensión del profesor Coto-Roque

\section{Desarrollo de evaluación interactiva en Forms}

La aplicación de Forms de Microsoft, permite realizar pruebas formativas interactivas, donde el profesor puede incluir elementos multimedia dentro de la prueba, para que el estudiante en tiempo real logre desarrollar su pensamiento creativo y analítico, esta herramienta de evaluación disruptiva puede integrar estrategias como el análisis de caso, prácticas programadas STEM y otras aplicaciones de evaluación formativa. Ver Figura 4.
Figura 4. Ejemplo de una prueba realizada en Forms

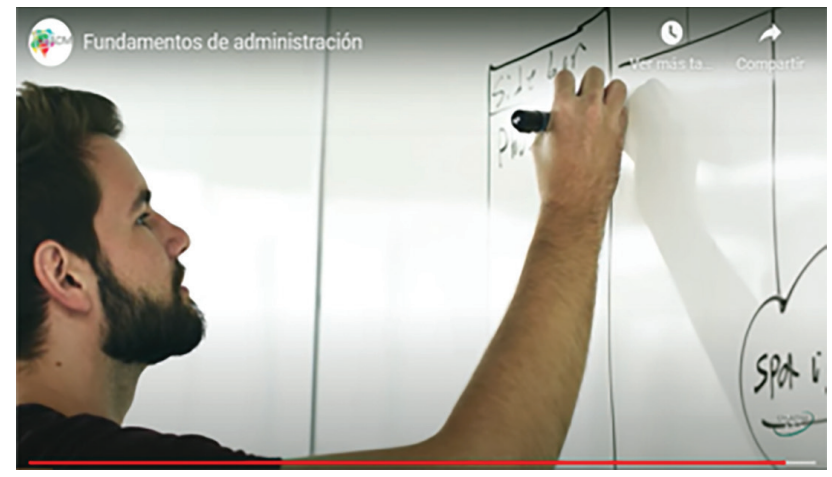

Fuente propia: Desarrollo e innovación Curricular

e. Estrategias de aprendizaje STEM en Google for Education Mind Mup. Los mapas mentales son estrategias disruptivas para mapear e internalizar aprendizajes significativos. La Universidad ha formado a sus profesores para promover la resolución de problema complejos, considerando la aplicación de Mind Mup, como Interfase para el desarrollo de mapas mentales. https://www.mindmup.com/

\section{f. Aprendizaje en realidad aumentada (RA)}

La realidad aumentada (RA) es la combinación de ambientes reales, a los cuales se incorpora información en formato digital con el fin de ampliar lo que nuestros sentidos captan sobre situaciones de la realidad. Esto se puede visualizar en una pantalla donde se mezclan la realidad captada por una cámara (en tiempo real) y la información virtual creada previamente y sincronizada a través de marcas (p. ej., tarjetas con dibujos o diagramas en blanco y negro) o por posicionamiento geográfico (vinculado al uso de internet). Esto se concreta en la siguiente descripción: una cámara enfoca el ambiente real, donde el usuario cuenta con una o más marcas, las cuales se programan para asociarse a determinadas imágenes, estáticas o animadas en 3D, por ejemplo, de un compuesto químico, planeta, célula, proceso, diagrama u otro contenido; sucesivamente, otros marcadores pueden ser asociados a otras imágenes. De esta forma, cuando el usuario alinea marcadores frente a una cámara, esta lo reconoce y los traduce, mostrando la imagen 3D asociada. Al utilizar más de un marcador a la vez, se puede visualizar una imagen/animación 3D nueva, que ilustra, por ejemplo, el surgimiento de un nuevo compuesto químico, un plano diferente para observar la geometría de un objeto, entre otros (Merino, 2014).

\section{EI papel del docente STEM como un asesor (Coaching)}

Hoy saber significa unir puntos y hacerlos converger en una realidad compleja y multifactorial. El acompañamiento o coaching aplicado a la docencia, se entiende como un proceso colaborativo, sistemático y orientado a resultados, 
enfocado a soluciones, en los cuales el docente facilita la mejora del rendimiento académico, la reducción de la deserción, el logro de las metas, el aprendizaje autodirigido y el crecimiento personal y profesional del estudiante. Se construye el aprendizaje a través de conversaciones y diálogo constante, el estudiante comparte situaciones concretas y el docente lo acompaña, construyendo un ambiente seguro, de confianza y libre de juicio, donde presentan sus dudas sin perjuicio y generan un cambio. El docente tutor es un facilitador que estimula relaciones asertivas.

Este modelo STEM de manera general, señala como aspectos deseables del profesor del Siglo XXI: excelente conocimiento del campo del saber pertinente, la constante inquietud por los temas pedagógicos y didácticos, el trabajo centrado en el aprendizaje del estudiante, la constante estimulación y motivación al estudiante para interesarse en los temas de estudio, la justa evaluación del desempeño, la capacidad para la selección de temas de estudio, la claridad en el aprendizaje y/o generación del conocimiento y el compromiso con su tarea de docencia.

\section{El papel del estudiante STEM}

Con la incorporación del modelo STEM en la Universidad, Fidélitas se produce un cambio en la secuencia didáctica, los estudiantes pasan de ser meros receptores del conocimiento a convertirse en productores y emisores, formando parte activa del proceso de enseñanza y aprendizaje, convirtiéndose en los verdaderos protagonistas del acto educativo.

En el marco actual, los estudiantes dejan de ser personajes que reciben una formación puntual que les capacita, para convertirse en profesionales activos que puedan responder a las demandas de la sociedad, éstos ven aumentada su autonomía, regulada a través de las estrategias de aprendizaje basadas en la competencia de aprender a aprender.

\section{Discusión}

- La Universidad Fidélitas de Costa Rica tiene una población estudiantil de Millennials y una nueva generación, llamada la generación Virtual (Gen $Z$ y Alpha), que exigen una innovación curricular que cumpla con sus expectativas profesionales y personales. Ante esta realidad la Universidad debe aplicar el modelo STEM, para responder a las necesidades prácticas, digitales y virtuales de las generaciones creativas del Siglo XXI ( $Y, Z$ y Alfa).

- Como se explicó, las principales universidades a nivel global utilizan el modelo STEM, que integran los conocimientos, habilidades, actitudes y valores, hacia la consecución de propósitos concretos para la vida personal y profesional de los estudiantes.
- Así las cosas, la Universidad Fidélitas considera que el modelo de aprendizaje STEM es un proceso didáctico, que impactará las acciones de innovación, emprendedurismo y cambio, utilizando los métodos de investigación y el pensamiento estratégico de diseño (Desing Thinking) para resolver los problemas de la sociedad contemporánea.

- $\quad$ En este marco de impacto académico y administrativo el modelo de aprendizaje STEM, promoverá espacios abiertos, a través de la fábrica de herramientas didácticas disruptivas, en la cual se desarrollan estrategias para potenciar las habilidades creativas en los estudiantes.

- Para los docentes que han participado en los talleres STEM, el modelo impacta los saberes, y es muy natural para los estudiantes de las nuevas generaciones aprender, evitando la fragmentación disciplinar $\mathrm{y}$ logrando la integración de contenidos significativos para la resolución de problemas, así la Universidad debe establecer un proyecto de transición de sus cursos tradicionales a cursos STEM, tomando en cuenta que el modelo de aprendizaje STEM, genera aprendizajes situacionales complejos, individuales y en grupo, y favorece la autonomía de los estudiantes, procurando el pensamiento complejo, la innovación y la aplicación de las tecnologías exponenciales, todo ello en coherencia con las actividades de evaluación formativa.

\section{Conclusiones}

- Continuar con la implementación del modelo de aprendizaje STEM, en el proceso de actualización de habilidades docentes de la Universidad, para aprovechar y potenciar todos los recursos académicos disponibles.

- Fabricar herramientas didácticas disruptivas que mejoren el proceso de aprendizaje, con el fin de ir transformando los cursos tradicionales a cursos STEM.

- Seguir implementando el proceso de virtualización flep learning o aula invertida, en el cual las nuevas tecnologías, principalmente las relacionadas con el acceso a materiales digitalizados y/o interactivos, faciliten la mediación con las generaciones creativas del Siglo XXI (Y, Z y Alfa). 


\section{Referencias}

Benito, A., y Cruz, A. (2016). Nuevas claves para la docencia universitaria en el espacio europeo de educación superior. Narcea S.A. ediciones.

Brodeur, D., Crawley E., Malmqvist, J. y Ostlund, S. (2007). Rethinking Engineering Education, The CDIO Approach. Springer.

CINDE. (2016). Necesidades de Capital Humano 2011-2016. Costa Rica.

Caprile, M. y Serrano, A. (2010). The move towards the knowledge-based society: a general approach. Gender Approach. Gender Work and Organization, 18 (1), 48-72. https://www.researchgate.net/publication/229921123 The_Move_Towards_the_Knowledge-based_Society_a_ Gender_Approach

Christensen, C. y Eyring, H. (2011). The Innovative University: Changing the DNA of Higher Education. Jossey-Bass.

Corominas, E., Tesouro, M., Capell, D., Teixidó, J., Pélach, J. y Cortada, R. (2006). Percepciones del profesorado ante la incorporación de las competencias genéricas en formación universitaria. Revista de Educación, 341, 301-336. http:// www.educacionyfp.gob.es/revista-de-educacion/numerosrevista-educacion/numeros-anteriores/2006/re341/re34114.html

Domènech-Casal, J. (2018). Aprendizaje Basado en Proyectos en el marco STEM. Componentes didácticas para la Competencia Científica. Revista de Educación Científica, 2 (2) 24-92. https://doi.org/10.17979/arec.2018.2.2.4524

ETH. Análisis de la estructura curricular y administrativa de la Universidad Escuela Politécnica Federal de Zürich https:// www.ethz.ch/de.html

Freire, P. y Faundez, A. (2010). Por una pedagogía de la pregunta. Crítica a una educación basada en respuestas a preguntas inexistentes. Siglo veintiuno.

Merino, S., Pino, S., Meyer, E. Garrido, J. y Gallardo, F. (2014). Realidad aumentada para el diseño de secuencias de enseñanza-aprendizaje en química. Educación Química, 26 (2), 94-99. https://www.sciencedirect.com/science/ article/pii/S0187893X15000051

MIT. Análisis de la estructura curricular y administrativa del Instituto Tecnológico de Massachusetts (Aspectos consultados en línea) http://web.mit.edu/

OCDE (2013). Programe for international Student Assesment (PISA).

Robinson, K. 2016. Creative Schools. The Grassroots Revolution That's Transforming Education. Penguin Books.

Tobón, S., Rial, A., Carretero, M. y García, J. (2006). Competencias, Calidad y Educación Superior. Editorial Magisterio.

Universidad de Harvard. Análisis de la estructura curricular y administrativa de la Universidad de Harvard (Aspectos consultados en línea) https://www.harvard.edu/

Universidad de Singapur. Análisis de la estructura curricular y administrativa de la Universidad de Singapur (Aspectos consultados en línea) http://nus.edu.sg /

Vázquez, J. (2011). Los caminos de Bolonia. Revista de docencia universitaria. Revista de Docencia Universitaria, 9 (3), 29-38. https://dialnet.unirioja.es/servlet/ articulo? codigo $=4019237$

Vilanova, N.(2017). Generación Z: Todo lo que necesitas saber sobre los jóvenes que han dejado viejos a los Millennials. Editorial Plataforma.

Villa, A. y Poblete, M. (2007). Aprendizaje basado en competencias. Una propuesta para la evaluación de las competencias genéricas. Universidad de Deusto, Bilbao. http://biblio.upmx.mx/textos/14633.pdf

Villardón-Gallego, L., Yániz, C., Achurra, C., Iraurgi, I. y Aguilar, M. (2013). Learning Competence in University: Development and Structural Validation of a scale of measure. Revista de Psicodidáctica, 18 (2), 357-374. https://ojs.ehu.eus/index.php/psicodidactica/article/ view/6470/6344

Villardón-Gallego, L (2015). Competencias genéricas en educación superior: Metodologías específicas para su desarrollo. Narcea Universitaria.

Sugata, M. (2013). The Ted Book. LLC. USA.

Yániz Álvarez de Eulate, C. (2007). Competencias en la Universidad. De la utopía a la pragmatopía. Nueva Época, (49), 4-10. https://www.researchgate.net/ publication/272166911_Competencias_en_la_ Universidad_De_la_utopia_a_la_pragmatopia

Santiago, R; Bergmann, J. (2018). Aprender al revés: Flipped Learning 3.0 Metodologías Activas en el aula. Ediciones Paidós

Yániz Álvarez de Eulate, C. (1). Planificar la enseñanza universitaria para el desarrollo de competencias. Educatio Siglo XXI, 24, 17-34. https://revistas.um.es/educatio/ article/view/151

Unidad de Currículum y Evaluación del Ministerio de Educación. (2019). Metodología de aprendizaje basado en proyectos. Unidad de Currículum y Evaluación del Ministerio de Educación. https://www.curriculumnacional. cl/614/articles-140166_recurso_pdf.pdf Revisar si es de internet la Fuente o de las bases de datos 